\title{
Fault tolerance reliability evaluation in multistage interconnection network
}

\begin{abstract}
Multistage interconnection networks (MINs) provide communication among processors, memory modules and other devices in parallel computer systems. These networks are designed to provide fast and efficient communication at a reasonable cost. The number of stages, interconnection topology, and network configuration differentiate the reliability of each network. Reliability is used to measure the system capability to transform information from input to output devices and is dependent on the reliability of its component. Researchers have previously attempted to evaluate the reliability of the interconnection network. This paper is an in-depth study concerning the evaluation of the reliability in multistage interconnection networks. The measurement includes the terminal reliability of the shuffle exchange network and extra group network.
\end{abstract}

Keyword: Reliability; Fault tolerance; Shuffle exchange network; Extra group network; Multistage interconnection network 